\title{
The fractal model of mechanical stress transfer in nanocomposites polyurethane/carbon nanotubes
}

\author{
G. V. Kozlov, I. V. Dolbin ${ }^{\dagger}$ \\ †i_dolbin@mail.ru
}

Kh. M. Berbekov Kabardino-Balkarian State University, 173 Chernyshevsky st., Nal'chik, 360004, Russia

In this communication effect of structure of nanofiller (carbon nanotubes) on transfer of applied to sample mechanical stress from polymer matrix to nanofiller was considered on example of nanocomposites polyurethane/carbon nanotubes. It was postulated that carbon nanotubes are formed in polymer matrix of nanocomposite ring-like formations, which are structural analogue of macromolecular coils of branched polymer chains and specific type of aggregation for carbon nanotubes in virtue of their low transverse stiffness and high anisotropy degree. Indicated formations structure can be characterized most precisely and physically strictly by its fractal dimension. It has been found, that transfer of mechanical stress in nanocomposite deteriorates at enhancement of indicated fractal dimension and at reaching of this dimension of value of parameter for surrounding Euclidean space nanofiller losses ability to reinfore matrix polymer owing to absence of mechanical stress transfer between its components. Thickness of interfacial layer in polymer nanocomposite is structural characteristic, defining efficiency of applied mechanical stress transfer. The using of alternative methodics for determination of radius of ring-like formations of carbon nanotubes shows enhancement of their number per one indicated formation at increasing of nanofiller contents. This effect results also to enhancement of fractal dimension of ring-like formation of carbon nanotubes. By analogy with "permeable" macromolecular coils of polymer chains this type of aggregation of carbon nanotubes is realized at fractal dimension of their formations of 1.50 and more. Indicated analogue application allows to estimate the Flory-Huggins interaction parameter, which proves to be higher essentially in comparison with polymer macromolecules.

Keywords: nanocomposite, stress transfer, ring-like formation, fractal dimension, reinforcement.

УДК 541.64: 546.26

\section{Фрактальная модель переноса механического напряжения в нанокомпозитах полиуретан/углеродные нанотрубки}

\author{
Козлов Г.В., Долбин И. В. ${ }^{\dagger}$ \\ Кабардино-Балкарский государственный университет им. Х. М. Бербекова, \\ ул. Чернышевского, 173, Нальчик, 360004, Россия
}

В настоящем сообщении рассматривается влияние структуры нанонаполнителя (углеродных нанотрубок) на перенос приложенного к образцу механического напряжения от полимерной матрицы к нанонаполнителю на примере нанокомпозитов полиуретан/углеродные нанотрубки. Постулируется, что углеродные нанотрубки образуют в полимерной матрице нанокомпозита кольцеобразные формирования, которые являются структурным аналогом макромолекулярных клубков разветвленных полимерных цепей и специфическим типом агрегации для углеродных нанотрубок в силу их низкой поперечной жесткости и высокой степени анизотропии. Наиболее точно и физически строго структуру указанных формирований можно охарактеризовать ее фрактальной размерностью. Обнаружено, что перенос механического напряжения в нанокомпозите ухудшается по мере увеличения указанной фрактальной размерности и при достижении этой размерностью величины параметра для окружающего евклидова пространства нанонаполнитель теряет способность усиливать матричный полимер из-за отсутствия переноса механического напряжения между его компонентами. Структурной характеристикой, определяющей эффективность переноса приложенного механического напряжения, является толщина межфазного слоя в полимерном нанокомпозите. Использование альтернативной методики определения радиуса кольцеобразных формирований углеродных нанотрубок показало увеличение их числа в одном указанном формировании по мере повышения содержания нанонаполнителя. Этот эффект также приводит к повышению фрактальной размерности кольцеобразных формирований углеродных нанотрубок. Аналогично «протекаемым» макромолекулярным клубкам полимерных цепей этот тип агрегации углеродных нанотрубок реализуется при фрактальной размерности их формирований 1,50 и более. Применение указанной аналогии позволяет оценить параметр взаимодействия Флори-Хаггинса для указанных нанотрубок, который оказался существенно выше по сравнению с полимерными макромолекулами.

Ключевые слова: нанокомпозит, перенос напряжения, кольцеобразные формирования, фрактальная размерность, усиление. 


\section{1. Введение}

Как хорошо известно [1], степень переноса приложенного к образцу механического напряжения от полимерной матрицы к нанонаполнителю определяет свойства полимерных нанокомпозитов, по крайней мере, механические. Этот показатель тесно связан с такими структурными и механическими характеристиками нанокомпозитов как относительная доля или толщина межфазных областей, уровень межфазной адгезии и прочность на сдвиг межфазной границы [2]. Как показали экспериментальные исследования с использованием современных методик (ультрамалоуглового рассеяния рентгеновских лучей, малоуглового рассеяния нейтронов) [3], углеродные нанотрубки в полимерной матрице нанокомпозита образуют кольцеобразные формирования, которые являются структурным аналогом макромолекулярных клубков разветвленных полимерных цепей, в силу своей низкой поперечной жесткости и высокой анизотропии. Эти кольцеобразные формирования являются фрактальными объектами, чье структурное состояние наиболее точно и физически строго описывается с помощью их фрактальной размерности $D_{f}[4]$. Поэтому в настоящем сообщении будет исследована зависимость степени переноса приложенного механического напряжения от полимерной матрицы к кольцеобразным формированиям углеродных нанотрубок (УНТ), структура которых характеризовалась их фрактальной размерностью, для нанокомпозитов полиуретан/углеродные нанотрубки.

\section{2. Эксперимент}

В настоящей работе выполнен теоретический анализ экспериментальных результатов, полученных авторами [5]. В качестве матричного полимера использован эластомерный полиуретан марки Morthane PS455-203 производства Huntsman Polyurethane. В качестве нанонаполнителя применялись однослойные углеродные нанотрубки (нановолокна) марки PRT-HT-19 производства Applied Science Inc., имеющие наружный диаметр $50-120$ нм и длину 10 мкм и более [5].

Образцы нанокомпозитов полиуретан/углеродные нанотрубки (ПУ/УНТ) получены смешиванием растворов компонентов при содержании УНТ 1-20 масс. \% в полярном растворителе (тетрагидрофуране) и последующим их приготовлением методом полива. Затем образцы сушились в вакуумной печи при температуре $323 \mathrm{~K}$ до постоянной массы. Полученные образцы имели толщину 0,5-2,0 мкм. Механические испытания выполнены на приборе Tinius Olson H10KS при скорости ползуна 6 мм/мин и температуре 293 K [5].

\section{3. Результаты и обсуждение}

Как отмечалось выше, степень переноса механического напряжения в полимерных нанокомпозитах определяет их свойства. В случае степени усиления $E_{n} / E_{m}$ эту зависимость можно описать следующим перколяционным соотношением [2]:

$$
E_{n} / E_{m}=1+11\left(B \varphi_{n}\right)^{1,7},
$$

где $E_{n}$ и $E_{m}-$ модули упругости нанокомпозита и матричного полимера, соответственно (отношение $E_{n} / E_{m}$ принято называть степенью усиления нанокомпозита), $B-$ безразмерный параметр, характеризующий степень переноса напряжения, $\varphi_{n}$ - объемное содержание нанонаполнителя.

Величину параметра $B$ можно определить в рамках макроскопических моделей с помощью следующего уравнения [6]:

$$
\frac{\sigma_{Y}^{n}}{\sigma_{Y}^{m}}=\frac{1-\varphi_{n}}{1+2,5 \varphi_{n}} \exp \left(B \varphi_{n}\right),
$$

где $\sigma_{Y}^{n}$ и $\sigma_{Y}^{m}-$ предел текучести нанокомпозита и матричного полимера, соответственно, а значения $\varphi_{n}$ приняты согласно данным работы [7].

В свою очередь, фрактальная размерность $D_{f}$ кольцеобразных формирований УНТ может быть определена с помощью уравнения [4]:

$$
R_{\mathrm{CNT}}=3,40 \varphi_{n}^{-1 /\left(d-D_{f}\right)},
$$

где $R_{\mathrm{CNT}}$ - радиус указанных кольцеобразных формирований, который дается в нанометрах, $d-$ размерность евклидова пространства, в котором рассматривается фрактал (очевидно, в нашем случае $d=3$ ).

Величину $R_{\mathrm{CNT}}$ в полимерной матрице нанокомпозита можно оценить использованием следующего соотношения [8]:

$$
b_{\alpha}=4,8\left(R_{\mathrm{CNT}}^{2}-0,022\right),
$$

где $b_{\alpha}-$ безразмерный параметр, характеризующий уровень межфазной адгезии на границе полимер-нанонаполнитель, который определяется с помощью следующего перколяционного соотношения [2]:

$$
E_{n} / E_{m}=1+11\left(c \varphi_{n} b_{\alpha}\right)^{1,7},
$$

где $c$ - постоянный коэффициент, равный $\sim 2,8$ для углеродных нанотрубок.

На Рис. 1 приведена зависимость $B\left(D_{f}\right)$ для нанокомпозитов ПУ/УНТ, которая показала линейный спад $B$ по мере роста $D_{f}$, т.е. компактизации кольцеобразных формирований УНТ. Аналитически эта зависимость описывается следующим уравнением:

$$
B=12,2-6,1\left(D_{f}-1\right) .
$$

Рассмотрим критические точки зависимости $B\left(D_{f}\right)$ на основе уравнения (6). При плотноупакованных кольцеобразных формированиях, имеющих евклидову структуру, т. е. при $D_{f}=d=3$, перенос напряжения от полимерной матрицы к углеродным нанотрубкам не реализуется, $B=0$ и согласно уравнению (1) $E_{n} / E_{m}=1,0$, т.е. углеродные нанотрубки теряют способность усиливать нанокомпозит. Другими словами, усиление нанокомпозитов реализуется только нанонаполнителем, имеющим фрактальную структуру. Для точечных объектов $D_{f}=0$, что наблюдается для одной из аллотропических форм углеродных нанотрубок (фуллеренов), величина $B$ максимальна и равна 18,3. Авторы [9] для нанокомпозитов полиамид-6/фуллерены $\mathrm{C}_{60}$ в случае неагрегированно- 
го нанонаполнителя получили величину $E_{n} / E_{m}=1,16$ при $\varphi_{n}=0,006$. Расчет согласно уравнению (1) при $B=18,3$ и $\varphi_{n}=0,006$ дает величину $E_{n} / E_{m}=1,265$, что хорошо согласуется с цитированным выше экспериментальным показателем. Этот пример демонстрирует сильное влияние структуры нанонаполнителя на степень усиления нанокомпозитов, а фрактальный анализ позволяет количественное описание этого эффекта. Так, переход от точечных $\left(D_{f}=0\right.$, фуллерены) к линейным $\left(D_{f}=1,0\right.$, ориентированные УНТ) приведет к снижению $E_{n} / E_{m}$ от цитированного выше значения до $~ 1,13$ при этой же величине $\varphi_{n}$.

Далее рассмотрим два вопроса, имеющих непосредственное отношение к рассматриваемой проблеме. Во-первых, относительную долю межфазных областей $\varphi_{p h}$ можно определить с помощью простого уравнения [8]:

$$
B=\varphi_{p h} / \varphi_{n} .
$$

Под межфазной областью (слоем) понимается слой полимерной матрицы, прилегающий к поверхности нанонаполнителя, и структурно отличающийся от указанной матрицы, например, по плотности упаковки, степени кристалличности и т. п. это приводит к изменению свойств межфазного слоя по сравнению с объемной полимерной матрицей. Напрмер, модуль упругости указанных слоев может существенно превысить (на порядок и более) соответствующий показатель для матричного полимера [10].

Оценки согласно уравнению (7) показали увеличение $\varphi_{p h}$ от 0,167 до 0,365 в интервале $W_{n}=1-20$ масс. \% нанонаполнителя для рассматриваемых нанокомпозитов, которое сопровождается снижением $B$ от 8,1 до 2,6 , хотя на первый взгляд должен наблюдаться противоположный эффект. Мы предположили, что увеличение $B$ обусловлено ростом не объемного содержания межфазных областей $\varphi_{p h}$, а увеличением толщины межфазного слоя $l_{p h}$.

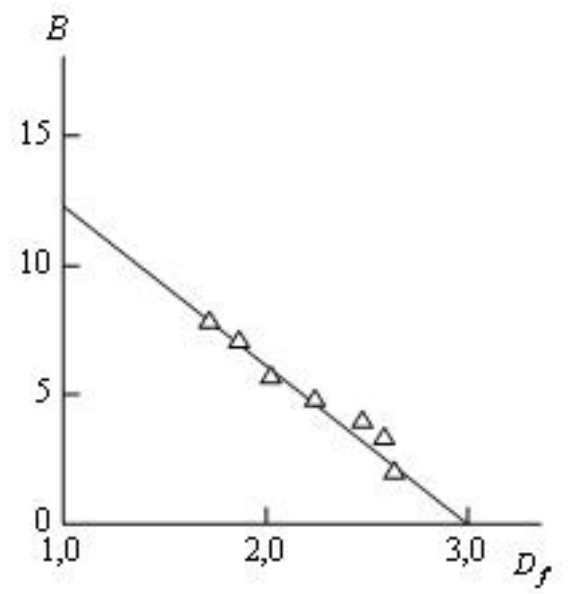

Рис. 1. Зависимость параметра $B$, характеризующего перенос механического напряжения, от фрактальной размерности $D_{f}$ агрегатов (кольцеобразных формирований) УНТ для нанокомпозитов ПУ/УНТ. Точки на графике соответствуют разному объемному содержанию $\varphi_{n}$ нанонаполнителя.

Fig. 1. The dependence of parameter $B$, characterized mechanical stress transfer, on fractal dimension $D_{f}$ of aggregates (ring-like formations) CNT for nanocomposites PU/CNT. The points on the graph correspond to the different volume contents of the $\varphi_{n}$ nanofiller.
Величину $l_{\text {ph }}$ можно определить с помощью следующего уравнения [10]:

$$
\varphi_{p h}=\frac{l_{p h}^{2}+2 r_{\mathrm{CNT}} l_{p h}}{r_{\mathrm{CNT}}^{2}} \varphi_{n},
$$

где $r_{\mathrm{CNT}}-$ радиус углеродной нанотрубки.

На Рис. 2 приведена зависимость $B\left(l_{p h}\right)$, которая показала ожидаемое повышение $B$ по мере роста $l_{p h}$ и аналитически описывается следующим уравнением:

$$
B=0,175 l_{p h} .
$$

где $l_{\text {ph }}$ дается в нм.

Следовательно, перенос приложенного напряжения и реализация усиления нанокомпозитов происходит только при ненулевых значениях $l_{p h}$. Уравнение (9) позволяет оценить предельное значение $B$ и, следовательно, максимальную степень усиления $E_{n} / E_{m}$ при фиксированной величине $\varphi_{n}$. Это предельное значение достигается в том случае, когда межфазные области заполняют все пространство полимерной матрицы между частицами (агрегатами частиц) нанонаполнителя, что соответствует условию:

$$
2 l_{p h}=S_{\mathrm{CNT}}
$$

где $S_{\mathrm{CNT}}-$ расстояние между поверхностями соседних углеродных нанотрубок, которое определяется согласно уравнению [1]:

$$
S_{\mathrm{CNT}}=2 r_{\mathrm{CNT}}\left[\left(\frac{\pi}{2 \sqrt{3} \varphi_{n}}\right)^{1 / 2}-1\right] .
$$

Например, при $\varphi_{n}=0,075\left(W_{n}=5\right.$ масс. \%) $S_{\mathrm{CNT}}=210 \mathrm{Hм}$ и $l_{\text {ph }}=105$ нм, а $B=18,4$. Тогда предельно возможное значение $E_{n} / E_{m}=20$, а соответствующая величина $E_{n}=200 \mathrm{MПа.}$ Отметим, что экспериментально полученная величина $E_{n}$ для этого нанокомпозита ПУ/УНТ равна 52 МПа [7].

Во-вторых, для определения радиуса кольцеобраз-

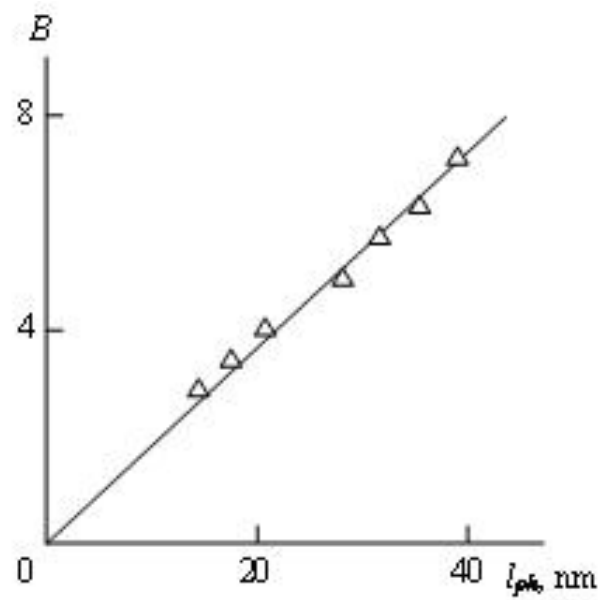

Рис. 2. Зависимость параметра $B$, характеризующего перенос механического напряжения, от толщины межфазного слоя $l_{p h}$ для нанокомпозитов ПУ/УНТ. Точки на графике соответствуют разному объемному содержанию $\varphi_{n}$ нанонаполнителя.

Fig. 2. The dependence of parameter $B$, characterized mechanical stress transfer, on thickness of interfacial layer $l_{p h}$ for nanocomposites $\mathrm{PU} / \mathrm{CNT}$. The points on the graph correspond to the different volume contents of the $\varphi_{n}$ nanofiller. 
ных формирований УНТ $R_{\mathrm{CNT}}$ можно использовать следующее уравнение [11]:

$$
\left(2 R_{\mathrm{CNT}}\right)^{3}=\frac{\pi N_{\mathrm{CNT}} r_{\mathrm{CNT}}^{2} L_{\mathrm{CNT}}}{\varphi_{n}},
$$

где $N_{\text {СNт }}$ - число углеродных нанотрубок в одном кольцеобразном формировании, $L_{\mathrm{CNT}}-$ длина нанотрубки.

На Рис. 3 приведена зависимость $D_{f}\left(N_{\mathrm{CNT}}\right)$ для рассматриваемых нанокомпозитов, которая оказалась линейной и аппроксимируется следующим уравнением:

$$
D_{f}=1,5+0,147\left(N_{\mathrm{CNT}}-1\right) .
$$

Следовательно, уравнение (13) демонстрирует, что увеличение числа углеродных нанотрубок в кольцеобразном формировании $N_{\mathrm{CNT}}$ приводит к его компактизации, характеризуемой повышением фрактальной размерности $D_{f}$. Возвращаясь к структурной аналогии указанных формирований и макромолекулярных клубков разветвленных полимерных цепей, отметим, что для последних было получено следующее соответствие [12]:

$$
D_{f}=1,5+0,45 \chi_{A B},
$$

где $\chi_{A B}-$ параметр взаимодействия Флори-Хаггинса.

Величину $\chi_{A B}$ для углеродных нанотрубок можно оценить из сочетания уравнений (12) и (13): оно варьируется в пределах 0,86-2,80, т. е. она существенно выше, чем для взаимодействия макромолекулярных клубков [13]. И в продолжение указанной выше аналогии отметим, что размерность $D_{f} \leq 1,50$ характеризует так называемые «протекаемые» макромолекулярные клубки, которые могут свободно пересекать друг друга, не взаимодействуя при этом. Поскольку из уравнения (13) следует, что и для УНТ при $D_{f}=1,50 N_{\mathrm{CNT}}=1,0$, то очевидно, что эта закономерность справедлива и в данном случае.

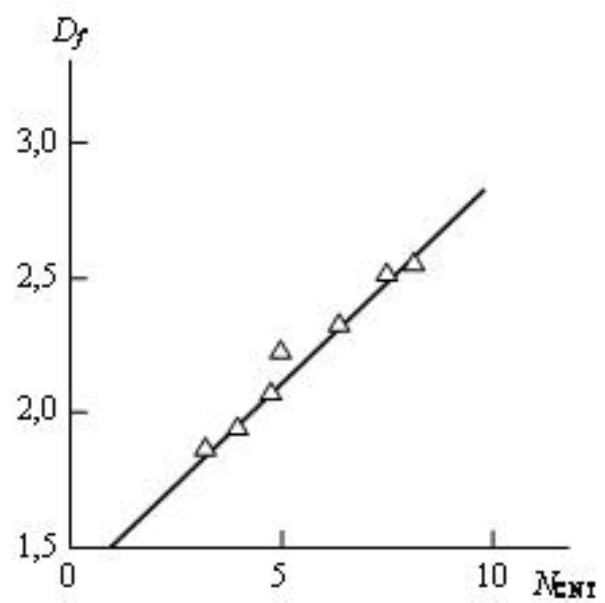

Рис. 3. Зависимость фрактальной размерности $D_{f}$ кольцеобразных формирований от числа углеродных нанотрубок в них $N_{\text {CNT }}$ для нанокомпозитов ПУ/УНТ. Точки на графике соответствуют разному объемному содержанию $\varphi$ нанонаполнителя.

Fig. 3. The dependence of fractal dimension $D_{f}$ of ring-like formations on number of carbon nanotubes in them $N_{\mathrm{CNT}}^{f}$ for nanocomposites PU/CNT. The points on the graph correspond to the different volume contents of the $\varphi_{n}$ nanofiller.

\section{4. Выводы}

Таким образом, результаты настоящей работы показали, что структура агрегатов (кольцеобразных формирований) углеродных нанотрубок, характеризуемая ее фрактальной размерностью $D_{f}$, оказывает решающее влияние на перенос приложенного напряжения в нанокомпозитах ПУ/УНТ - чем меньше $D_{f}$, тем эффективнее перенос механического напряжения. Структурным фактором, контролирующим перенос механического напряжения, является толщина межфазного слоя. Усиление полимерных нанокомпозитов реализуется только фрактальными наночастицами (агрегатами наночастиц), чья армирующая способность повышается по мере снижения размерности исходного нанонаполнителя. Увеличение числа углеродных нанотрубок в кольцеобразном формировании (агрегате) приводит к его компактизации, характеризуемой повышением фрактальной размерности.

\section{Литература/References}

1. B. Fiedler, F. H. Goijny, M. H. G. Wichman, M. C. M. Nolte. Composites Sci. Tech. 66, 3115 (2006), DOI: 10.1016/j. compscitech.2005.01.014

2. A. K. Mikitaev, G. V. Kozlov. Fizika i khimiya obrabotki materialov. 4, 65 (2015). (in Russian) [А.К. Микитаев, Г. В. Козлов. Физика и химия обработки материалов. 4, 65 (2015)]

3. D.W. Schaefer, R.S. Justice. Macromolecules. 40, 8501 (2007), DOI: 10.1021/ma070356w

4. A. K. Mikitaev, G. V. Kozlov. Doklady Physics. 60, 203 (2015), DOI: 101134/5102833581505002X

5. H. Koerner, W. Liu, M. Alexander, P. Mirau, H. Dowty, R.A. Vaia. Polymer. 46, 4405 (2005), DOI: 10.1016/j. polymer.2005,02.025

6. B. Pukanszky. Composites. 21, 255 (1990), DOI: 10.1016/0010-4361(90)90240-W

7. D.W. Schaefer, J. Zhao, H. Dowty, M. Alexander, E. B. Orler. Soft Mater. 4, 2071 (2008), DOI: 10.1039/ b805314f

8. Yu. G. Yanovsky, G. V. Kozlov, Z. M. Zhirikova, V.Z. Aloev, Yu. N. Karnet. Nannomechanics Sci. Techn.: Intern. J. 3 (2), 99 (2012), DOI: 10.1615/NanomechanicsSciTechnol. Int.J.v.3.i.2.10

9. V. V. Zuev, Yu. G. Ivanova. Polymer Science. Series A. 53, 424 (2011), DOI: 10.1134/S0965545X11050129

10. J. N. Coleman, M. Cadek, K. P. Ryan, J. B. Nagy, W. J. Blau, M.S. Ferreira. Polymer. 47, 8556 (2006), DOI: 10.1016/j. polymer.2006.10.014

11. B. Bridge. J. Mater. Sci. Lett. 8, 102 (1989), DOI: 10.1007/ BF00720265

12. G.V. Kozlov, I. V. Dolbin, G.E. Zaikov. The Fractal Physical Chemistry of Polymer Solutions and Melts. Toronto, New Jersey, Apple Academic Press. (2014) 316 p.

13. V.P. Budtov. Physical Chemistry of Polymer Solutions. St. Peterburg, Khimiya (1992) 384 p. (in Russian) [В.П. Будтов. Физическая химия полимерных растворов. СПб., Химия. 1992. 384 с.] 